\title{
Design of Low Cost Bio-impedance Measuring Instrument
}

\author{
Rajesh Birok, Rajiv Kapoor \\ Dept. of Electronics \& Communication Engineering \\ Delhi Technological University Delhi, India
}

\begin{abstract}
It is a well-established fact that the electrical bioimpedance of a part of the human body can provide valuable information regarding physiological parameters of the human body, if the signal is correctly detected and interpreted. Accordingly, an efficient low-cost bio-electrical impedance measuring instrument was developed, implemented, and tested in this study. Primarily, it is based upon the low-cost component-level approach so that it can be easily used by researchers and investigators in the specific domain. The measurement setup of instrument was tested on adult human subjects to obtain the impedance signal of the forearm which is under investigation in this case. However, depending on the illness or activity under examination, the instrument can be used on any other part of the body. The current injected by the instrument is within the safe limits and the gain of the biomedical instrumentation amplifier is highly reasonable. The technique is easy and user-friendly, and it does not necessitate any special training, therefore it can be effectively used to collect bio-impedance data and interpret the findings for medical diagnostics. Moreover, in this paper, several existing methods and associated approaches have been extensively explored, with in-depth coverage of their working principles, implementations, merits, and disadvantages, as well as focused on other technical aspects. Lastly, the paper also deliberates upon the present status, future challenges and scope of various other possible bio-impedance methods and techniques.
\end{abstract}

Keywords-Noninvasive; bio-electrical; Impedance; bioimpedance; bio-medical; instrumentation

\section{INTRODUCTION}

Extensive research is going on in the field of Bio-Medical Instrumentation. Researchers and investigators in this field are striving hard to find out new ways and methods for diagnosis and measurement of health parameters for the welfare of the mankind. There are two types of techniques for measuring biomedical signals namely non-invasive and invasive techniques. Non-invasive techniques are more suitable than the invasive ones if sufficient accuracy can be achieved using them. Whether, it is animal and plant cells or tissues, these are always made up of three-dimensional arrangement of cells and tissues. Therefore, human body is a complex biological structure and system, which is also made up of billions of cells and tissues arranged in 3-D formation [1]. The biological cells and tissues of both animals and plants floats in ECF which is known as Extra-Cellular Fluids. This ECF comprises Intra-Cellular Fluids (ICF) and Cell Membranes (CM) which may be with or without cell wall. When biological cells and tissues are subjected to the external electrical stimulus they respond and produces a complex bio-electrical impedance or simply known as bio-impedance. This bio-impedance is highly frequencydependent [2], [3].
Accordingly, frequency response of bio-impedance of cells and tissues of humans is greatly affected by physiological and physiochemical composition and structure of these cells and tissues. Moreover, it also changes from person to person. As a result, learning about cell and tissue anatomy and physiology through biological cell and tissue bio-impedance analysis will be a valuable resource. Therefore, it has been found that studying complex bio-impedance of biological cell and tissues is a useful method for non-invasive physiological and pathological investigations. As we know that the bio-electrical impedance of a biological cells or tissues is dependent on the signal frequency, however, multifrequency application may also be used for non-invasive diagnostics and medical investigations, so as to determine their physiological or pathological behavior or even properties. There are numerous Non-invasive bioimpedance techniques such as BIA (Bio-Impedance Analysis), EIT (Electrical Impedance Tomography), IPG (Impedance Plethysmography), ICG (Impedance Cardiography), etc. The bio-impedance measurement technique proposed in this research paper, is a low-cost, efficient, and effective non-invasive diagnostic technique.

\section{LITERATURE SURVEY}

Impedance offered by a living tissue is known as bioimpedance. The broad variability of Cole parameters makes it difficult to use bio-impedance to distinguish animal and plant tissues. [4] defines a novel electronic procedure for distinguishing fruit or vegetable tissue. This system uses a custom-built electrode pair to compute bio-impedance and Cole parameters covering a wide range of frequencies from $1 \mathrm{~Hz}$ to $1 \mathrm{MHz}$ [4]. However, impedance of human cell and tissue consists of resistive and capacitive components [5], [6]. It's determined by injecting an alternating current in the cell or tissue and then measuring the output voltage across it. The Linear Time-Varying (LTV) bio-impedance is measured with a specified precision using stepped-sine excitations, as given in [7], but it is susceptible to temporal distortions affecting the data, which limits the device's temporal bandwidth and sets the data accuracy. Current source and voltage sensing circuit are the essential blocks in the instrument. Several authors have successfully designed current sources operating up to few hundreds of $\mathrm{kHz}$ [8]. Paul Annus et.al. [9] have systematically analyzed the design of a current source using transfer function approach and they have measured the load impedance using load in loop method or configuration. The voltage sensing circuit consists of amplifier, demodulator and low pass filter. The use of instrumentation amplifier for bioimpedance measurements has been analyzed by Areny and Webster [10]. The measurement technique has been used in a 
number of applications such as calculating Total Body Water (TBW), calculating Intracellular Fluid (ICF) and extracellular fluid (ECF) [6] Electrical Cardiometry [11], Skin Water Content, Impedance Imaging (Tomography), Ablation Monitoring and measurement of Respiration Rate [12]. The technique also has the potential to be used in biometrics [13]. Body Composition Assessment, Transthoracic Impedance Pneumography, Electrical Impedance Tomography (EIT), and Skin Conductance are examples of bio-impedance applications that are described and analysed in [14]. [15] looked into the possibility of non-invasively tracking blood glucose levels using bioimpedance data, which would allow for more regular testing and better diabetes management and monitoring. The bioimpedance measurement is not a new technique in biomedical diagnostic techniques but research is still going on to make this technique a standard procedure for diagnosis of a particular disease. For this purpose, it is required that a large amount of data be collected for a particular disease, for a particular environmental society and analysis to be done, correlating the disease with the signal recorded. To attain this objective, we have designed a low-cost instrument using common analog signal processing blocks, which gives an accurate recording of the bio-impedance signal.

\section{BACKGROUND}

\section{A. Basics and Origin of Bio-impedance}

Bio-impedance is a passive electrical property that describes a biological cell or tissue's ability to obstruct (oppose) the flow of electrical current through it. The reaction to electrical excitation (current or potential) applied to biological tissue is used to determine bio-impedance. The same or other electrodes applies the excitation signal and picks up the reaction in bio-impedance measurements, then charge conversion takes place from electronic to ionic charge and vice versa [7]. Simply, the ratio of voltage (V) to alternating current (I) is known as electrical impedance (Z). Since, Direct Current (DC) is quite hazardous to humans, therefore, it is never used for any experimentation on humans. In fact, Alternating Current (AC) is more preferable choice for such type of applications. The calculated or observed bio-impedance ( $\mathrm{Z}$ ) is highly influenced by the Resistive (R), Capacitive (C), and Inductive (L) parts of the cells and tissues. The bio-impedance $(\mathrm{Z})$ is given by using the modulus $\mid \mathrm{ZI}$ and the phase change. Since, bio-impedance (Z) is a complex function or parameter, therefore its Resistance (R) is the real part and whereas, the Capacitance Reactance $\left(\mathrm{X}_{c}\right)$ is the imaginary part. As the ICF, CM, and ECF are made of dissimilar materials with nonidentical electrical properties, therefore every cell and tissue components react differently to the applied AC signal. As we know that ICF and ECF are made up of ionic solution which is highly conducting in nature, thus it provides low resistance path to the applied AC signal [16]. The CM are composed of lipid bilayers which are electrically nonconducting and inserted between two layers of conducting proteins. This sandwiched structure, produces a capacitive reactance $\left(\mathrm{X}_{c}\right)$ to the applied AC signal [17], [18]. Due to this, biological cells and tissues produces a complex bio-impedance $(\mathrm{Z})$ which can be considered as overall response to an applied AC signal [2], [3]. Thus, bio-impedance (Z) is a complex function depends upon cell and tissue composition and structure, health of person and applied AC signal frequency. Moreover, it also changes with measurement direction, from one subject to the other subject and even within the tissue itself.

The human body composition comprises, water (64\%), protein $(20 \%)$, fat (10\%), and minerals $(5 \%)$ and starch (1\%). The human body mass is mainly due to $\mathrm{O}(65 \%), \mathrm{C}(18 \%)$, and $\mathrm{H}$ $(8 \%)$. The majority of muscles are made up of protein whereas, majority of bones are made up of minerals [19]. The bioimpedance is proportional to Total Body Water (TBW), which contains Intra-Cellular Water (ICW) and Extra-Cellular Water (ECW). Body water, body fat, and body muscle have different impedance values according to the amount of presence of water in these. Thus applied AC signal pass through paths that contain more water as it provides high conductivity [20]. The physiological, morphological, pathological settings and also applied AC signal frequency, all these affect and influence cells and tissues and their electrical properties [21], [22]. The biological cells and tissues may have active (endogenous) or passive (exogenous) electrical properties, depending on the type of source of applied AC signal. The bio-electric signals from the heart known as electrocardiograph (ECG), signals from the brain known as electroencephalograph (EEG), whereas electromyograph (EMG) signals from the muscles are few examples of active properties (bio-electricity) produced by ionic activities within cells and tissues (typical of nerve cells). Passive properties are generated by simulating them with an external electrical excitation source [23], [24].

The extracellular fluids surround all cells with membranes in biological tissues. The main constituents of Extra-Cellular Fluid (ECF) are fluid component of the blood known as plasma and the other one is Interstitial Fluid (IF) which surrounds all cells that are not in blood. The extracellular space is the part of a multicellular organism outside the cells, whereas intracellular space is within the organism's cells. The cell membranes separate extracellular spaces and intracellular space thus producing two electrically conducting compartments known as extracellular media and intracellular media. The resistive pathways are provided by ECF and intracellular fluids (ICF). Due to its insulating design and structure, the lipid bilayer cell membrane is very-very thin measuring approximately 6-7 $\mathrm{nm}$. This lipid bilayer cell membrane is semi-permeable, due to which it has a high capacitance and which produces capacitive reactance [25], [26], [27]. Although biological cells and tissues may have inductive properties, inductance is much lower at low frequencies than resistance and reactance, so it is often overlooked [28]. Thus, biological cells or tissue's complex bioimpedance is the contributions from both frequency-dependent capacitance and conductance [21], [29], [30], [31], [32], [33]. Bio-electrical impedance often differs from one tissue to the next, as well as from one subject to the next. The complex bioelectrical impedance is affected by changes in cell and tissue composition and structure, and even health condition or status of the subject [5], [6].

\section{B. Frequency Response of Bio-impedance}

The anatomical, physiological, and pathological state of biological cells and tissues determine the bio-impedance frequency response. Therefore, the bio-impedance study can provide much more information related to the anatomy and physiology of a cell or tissue. Since the bio-impedance response is a variable of signal frequency, therefore bio-impedance analysis 
with multifrequency inputs can give detailed cell or tissue attributes information, that can help greatly in cell or tissue specifications. Furthermore, the applied ac signal frequency also drastically affects it [2], [3]. Thus, the bio-impedance of cells or tissues and their frequency response is greatly affected not only by the composition and structure of these cells and tissues in the given physiological and physiochemical setting but also by the applied signal frequency. The few bio-impedance analysis techniques which makes use of lumped estimation of the bio-impedance values of the cell or tissue samples are BIA, IPG, and ICG etc. Bio-Electrochemical Impedance Spectroscopy (EIS) measures and analyses bio-impedance at different frequencies. Thus, EIS provides not only a lumped approximation of the cell or tissue sample's bio-impedance values at relatively higher frequency (generally $50 \mathrm{kHz}$ ), but also the details required to have better understanding of the many complex bio-electrical phenomena such as dielectric dispersions and relaxation.

The bio-impedance measurement can be broadly divided into two categories, namely, "single-tone" signals and "multitone" signals measurements. The analysis of "single-tone" signals is very straightforward, but measurements take longer, whereas use of a multi-tone signal allows for simultaneous coverage of the entire frequency spectrum. However, use of a multi-tone signal may result in an algorithm which can be more complex for analysis purpose [34]. Moreover, especially the dielectric properties of the object determine the required frequency range for bio-impedance measurements, which typically spans 3 to 4 decades in the $\mathrm{kHz}$ to $\mathrm{MHz}$ range. In general, wider range of frequencies improves fitting accuracy but at the cost of complicating measurements. Furthermore, (SNR) of measured signals also effects the fitting accuracy [35].

\section{Types of Electrode Configurations}

When an alternating current is used in bio-impedance testing, the electrode displays a frequency-dependent Electrode Polarisation Impedance (EPI) at the contact point with the tissue or solution as the case may be. Any change in the electrode material that is in contact with the tissue or solution also affects the magnitude and as well as the phase of the electrode impedance. As a result, the total calculated impedance of the system is equal to the sum of the EPI and impedance of the tissue/solution [25]. Finally, the impedance depends upon type of electrode used, electrode material used, the applied signal amplitude and also on its size, shape and structure [36]. In order to measure bio-impedance, we need at least two electrodes for the electrical current to pass through the closed circuit [25]. Therefore, bio-impedance measurements are performed with two or four electrodes. In both methods, the red coloured electrodes depicted in Fig. 1 are known as input or excitation electrodes. Basically, these are the current or driving electrodes. On the other hand, blue coloured electrodes also depicted in Fig.1 are known as voltage/sensing or output electrodes used to determine output signal which is frequency dependent. It should be observed that bio-impedance measurements can be inaccurate due to factors such as movement and improper electrode placement [36]. Bio-impedance measurements are typically done with gel electrodes to minimise electrode-skin impedance. The usability of dry electrodes is studied in [37] because this type of electrode is not suitable in many measurement environments. For bioimpedance measurement, there are two kinds of electrode configurations. According to its name the two-electrode system or setup depicted in Fig.1(a) for measurement of impedance makes use of two electrodes only. Thus, the current signal injection or current-carrying as well as voltage measurement or voltage pickup are done with the same electrodes. For unipolar measurements, electrode configuration with quite large and small electrodes can also be used [38].

The two-electrode technique suffers from contact impedance due to polarisation impedance at the electrodes' surfaces and the measured signal also covers the contact impedance due to voltage drop [25]. While, analysing the measured signal, the polarisation impedance should be considered and removed in the output signal [39], [40]. Overall, the results obtained using this method are interesting, however they do not provide an accurate signal on the electrode's surface [41]. In four electrode configuration or setup, two independent pair of electrodes are used for current injection and detecting changes in voltage or voltage measurements [39], [42]. In this system, the input that is constant amplitude current signal is injected using the outer electrodes. These electrodes are also known as current or driving electrodes depicted in red colour in Fig.1(b). The output voltage signal produced which is frequency dependent is measured at two points within the current electrode. These electrodes are also known as voltage or sensing electrodes depicted in blue colour in Fig. 1(b). In this configuration, as the distance between electrode pairs was increased, the magnitude of the measured impedance decreased [25], [43]. A number of factors influence the effect of electrode polarisation impedance which includes electrode content, size, measuring frequency, sample impedance, and so on. The main advantage of four electrode configuration over its counterpart two electrode configuration is that the voltage or sensing electrodes do not carry current, due to this it eliminates polarisation impedance. Thus, at the connecting surface of electrode with tissue or electrolyte the influence of contact impedance is greatly decreased. Therefore, use of this method is a common and popular practice to lower the effect of EPI [44]. Furthermore, four electrode configuration measurements are more sensitive and accurate.

\section{PROPOSED METHOD}

The instrument designed consists of power supply, current source, voltage sensing unit and data acquisition system (DAS). Fig. 2 depicts the general block diagram of the measuring instrument. The current source circuit is used to generate a sinusoidal current of amplitude in the range of $600 \mu \mathrm{A}-800 \mu \mathrm{A}$ and a frequency of $50 \mathrm{kHz}$ [4]. The voltage sensing unit is used to amplify and remove the high frequency components from the signal sensed by voltage sensing electrodes. The signal is then given to the data acquisition system. The contact surface dimensions of all test electrodes are the same, and the carrier is a circular printed circuit board. To compare the electrodes' characteristics, the electrode-skin impedances are measured under a variety of signal frequencies, contact durations, contact pressures, positioning positions, and subjects. All measurements are often done with silver/silver chloride $(\mathrm{Ag} / \mathrm{AgCl})$ dry gel electrodes for contrast. [37] The instrument has four $\mathrm{Ag} / \mathrm{AgCl}$ electrodes, two on the outside and two on the inside, 


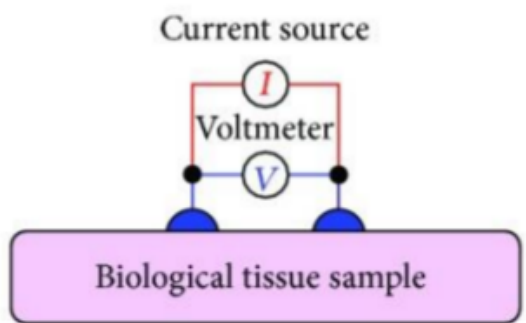

(a)

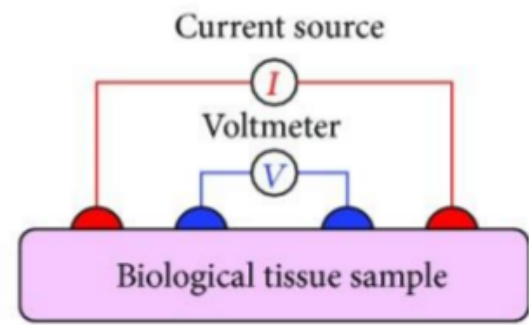

(b)

Fig. 1. Bio-impedance Measurement using: (a) Two- Electrode Method, (b) Four-electrode Method.

which are used as current electrodes and voltage electrodes, respectively. This type of arrangement is known as a tetra polar arrangement. Such an arrangement has been implemented by $\mathrm{J}$. J. Wang et.al. [45] to use forearm impedance plethysmography for monitoring cardiac pumping function.

\section{A. Waveform Generation}

This is the first stage of the instrument and also the most critical one. All the parameters for this stage are very important as far as subject's safety and the output of the device is concerned. As this is the first stage any noise added at this stage will get amplified in the subsequent stages. A good waveform generator should have a single frequency output with very low value of total harmonic distortion and should have a very low frequency drift. A Wein bridge oscillator can be used to generate a sinusoidal waveform [46]. The oscillator is designed for a frequency of $50 \mathrm{kHz}$ as this frequency is widely accepted in clinical use as a standard [47]. Among all types of voltage source generators, an oscillator produces the output with the most stable frequency as compared to other sources. Wein bridge oscillator is implemented using an op-amp, which gives a stable output with a single frequency for a particular combination of resistors and capacitors. Proper limiter circuit is also used in the circuit to keep the poles of the oscillator on the imaginary axis. One major problem with using Wein bridge oscillator is that the instrument designed cannot be used for bio-impedance spectroscopy.

A comparator, integrator and wave shaping circuit can be used to generate square, triangular and sinusoidal waveform of variable frequency [48]. The comparator and integrator circuit used together generate square and triangular waveforms. Triangular waveform is shaped using a shaping circuit to generate sinusoidal waveform. The maximum frequency achieved by this circuit using 741 op-amp is $26 \mathrm{kHz}$. This method can be used for excitation in lower frequency range but lower frequencies are not used in bio-impedance spectroscopy as electrodes get polarized at lower frequencies. It has also been observed during our laboratory experiments that there was no biomodulation obtained in the output signal when a current of frequency less than $20 \mathrm{kHz}$ was injected into human body. The instrument designed uses a monolithic integrated circuit ICL8038, to produce high accuracy sine, square and triangular waveforms [49]. The frequency can be selected externally from $10 \mathrm{kHz}$ to $100 \mathrm{kHz}$ using a potentiometer. The output of ICL8038 is stable over a wide range of temperature and supply variations. The total harmonic distortion for ICL8038 varies from $1 \%$ to $2 \%$ depending on the model selected.

\section{B. V To I Convertor}

The current driver is one of the most important sub circuit for the measurements of bio-impedance. The current driver can easily work over a fairly wide range of impedance and frequency. The main requirements of a current driver are high output impedance, short phase delay and minimal harmonic distortion. Depending on whether they are open loop or closed loop, these are categorized into two groups. The features of each design are described [50].

The voltage waveform generated using 8038 is converted into current, which is injected into human body. The current generated is within the safe limits $(600 \mu \mathrm{A}-800 \mu \mathrm{A})$. The $\mathrm{V}$ to I converter is intended to run in the frequency range of 10-100 $\mathrm{kHz}$.

The main requirement for a current source is that it should supply a constant amount of current irrespective of the impedance of the load connected to it. When the output impedance of the current source is much greater than the load impedance then the current through the load is maintained constant regardless of the load value. F. Seoane et.al have analyzed Howland circuit as V to I converter [51]. In this instrument Howland circuit with buffer is used for voltage to current conversion. Fig. 3 is the circuit diagram for voltage to current converter. The buffer stage increases the output impedance of the $\mathrm{V}$ to I converter. The input impedance of the circuit is around $20 \mathrm{k} \Omega$. Fig. 4 depicts the simulation results for circuit of Fig.-3. The resistance $\{R\}$ in the Fig. 3 represents the load to which current would be injected. The human body impedance is in the range $1 \mathrm{k} \Omega-3 \mathrm{k} \Omega$. In simulation the resistance $\{R\}$ is varied from $0.1 \mathrm{k}$ to $5 \mathrm{k}$ in steps of $100 \Omega$. It is verified using the simulation results that the current through the load is almost constant (10 $\mu \mathrm{A}$ variation) irrespective of the load resistance.

\section{Instrumentation Amplifier}

The voltage sensed from the section of human body is in range of millivolts. The input signal is amplified using a difference amplifier. A single op-amp can also be used as a difference amplifier but due to its low input impedance it cannot be used. The input impedance of the single op-amp difference amplifier can be increased by introducing a buffer at each of the inputs of the amplifier and instead of using a unity gain follower one can also have some gain from the first 


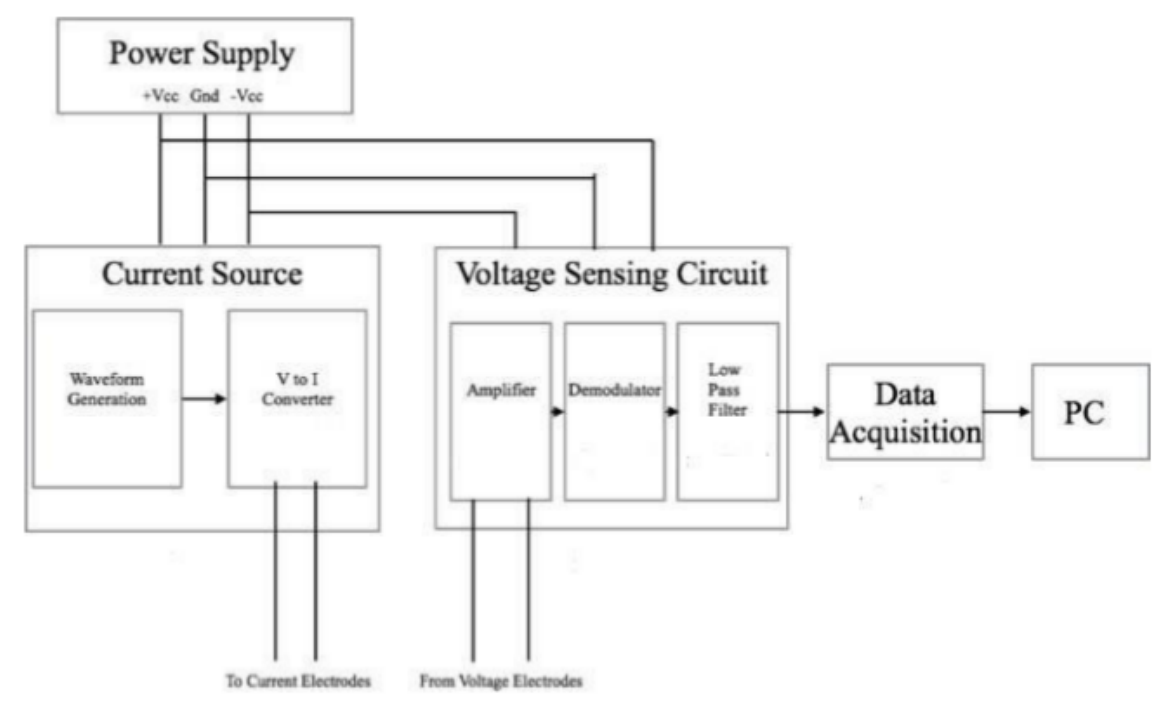

Fig. 2. General Block Diagram of Measuring Instrument.

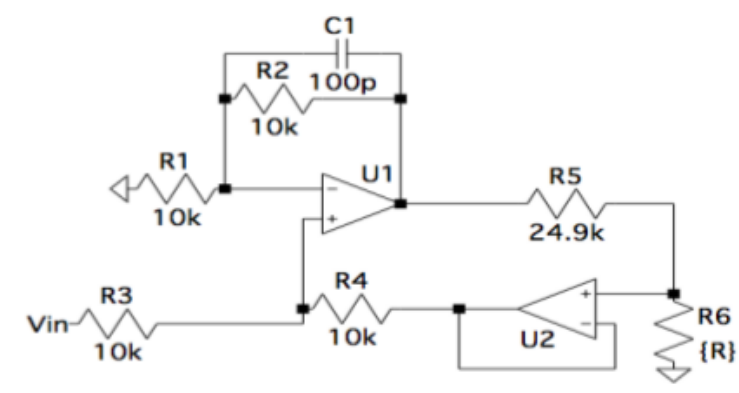

Fig. 3. Circuit Diagram for Voltage to Current Converter.

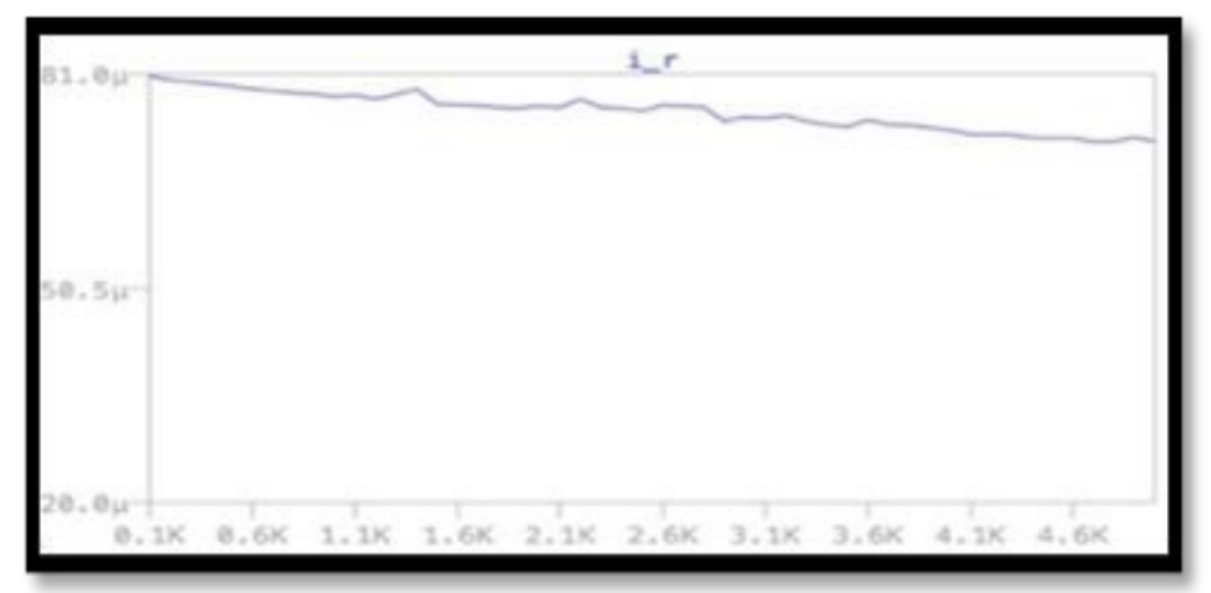

Fig. 4. Variation of Current Through $\{R\}$ with Respect to Change in Value of Load Resistance, $y$-axis is the Current Through the Load and $x$-axis is the Impedance of the Load Connected to the V to I Converter. 
stage. This leads to the circuit of instrumentation amplifier as shown in Fig. 5. The instrumentation amplifier has high CMRR and high input impedance and has been very effectively used in biomedical applications such as EEG and ECG.

The analysis for instrumentation amplifier has been given by Adel S. Sedra and Kenneth C. Smith [1] and its use as a biopotential amplifier has been analyzed by Nagel J.H [52]. The gain for the instrumentation amplifier is given below.

$$
\text { gain }=\frac{R_{4}}{R_{3}}\left(1+\frac{R_{2}}{R_{1}}\right)
$$

The signal from the electrodes is given as input to the instrumentation amplifier through its two terminals. The gain of the amplifier is $26.44 \mathrm{~dB}$. The first two op-amps are used for gain and the third operational amplifier is used for common mode rejection. In the circuit designed $R_{4}=R_{3}$ and $R_{2} / R_{1}$ is equal to 20 . The amplifier's input impedance is equal to the input impedance of the operational amplifier. Frequency response analysis of the instrumentation amplifier depicts that the phase and gain are constant in the desired frequency range. Fig. 6 depicts the frequency response of the instrumentation amplifier in which the continuous plot is the magnitude plot and dotted plot is the phase plot.

\section{Demodulator}

The signal obtained from the human body is an amplitude modulated signal, where the carrier is the current waveform that we have introduced into the human body. In this case the high frequency carrier is a sinusoidal waveform with a frequency of $50 \mathrm{kHz}$ and the modulating bio-impedance signal is low frequency signal with frequency components less than $50 \mathrm{~Hz}$. Webster and Tompkins [53] have suggested use of full wave rectifier for demodulation. The modulating signal can be extracted using a simple envelope detector circuit as shown in Fig. 7. Asynchronous demodulation has been used so that square and triangular waveform excitation can also be used in addition to the sinusoidal waveform.

For the first cycle diode is forward biased and it charges the capacitor to the first peak value. The charging time constant should be such that the capacitor voltage follows the input signal.

$$
\tau \text { charging }<<\frac{1}{f_{c}}
$$

where $\mathrm{f}_{c}$ is the frequency of the carrier. The charging times constant depends on source resistance $\mathrm{R}_{s}$, forward bias diode resistance $r_{d}$ and capacitance $C_{1}$.

$$
\begin{gathered}
\tau \text { charging }=\left(\left(R_{s}+r_{d}+R_{2}\right) \| R_{1}\right) C_{1} \\
R_{s}+r_{d}+R_{2}<<R_{1} \\
\therefore \quad \tau \text { charging }=\left(R_{s}+r_{d}+R_{2}\right) C_{1}
\end{gathered}
$$

When the input signal drops, diode becomes reverse bias (as capacitor is charged to a higher voltage) and the capacitor voltage remains at the initial level. During this time (when diode is reverse bias) the capacitor discharges through the resistor $\mathrm{R}_{1}$.

$$
\tau \text { discharging }=R_{1} C_{1}
$$

The discharging time constant should be large so that the capacitor discharges slowly but it should not be so large that it is unable to trace the low frequency modulating signal. The discharging time constant should follow the following relation:

$$
\frac{1}{B} \gg \tau \text { discharging } \gg \frac{1}{f_{c}}
$$

Where B is the bandwidth of the low frequency bio-impedance signals. For the calculation of the values of resistances and capacitance, value of B is taken equal to $50 \mathrm{~Hz}$. The diode used in the circuit is forward biased when the incoming voltage is greater than $0.7 \mathrm{~V}$, therefore the low voltage signals cannot be detected. Taking into account this problem related to cut off voltage of diode, a precision diode [46] is used in this circuit in place of normal diode. A precision diode is an operational amplifier with a diode in the negative feedback followed by a diode whose anode is connected at the output pin of the opamp. The cutoff voltage for a precision diode is approximately equal to zero volts. Fig. 8 is the circuit for precision diode. The output of the demodulator is given to the low pass filter stage through a buffer. Improved output buffering and peak detector gain greater than unity is achieved with an output voltage follower. This leads to circuit of precision envelope detector in Fig. 9. The precision envelope detector is much more accurate than the simple envelope detector as the voltages below $0.7 \mathrm{~V}$ are also detected by this circuit. Droop due to detector diode leakage can be removed through the use of bootstrapping feedback that holds detector diode bias at zero when the diode is not conducting.

\section{E. Low Pass Filter}

The signal from the demodulator contains high frequency components, which needs to be filtered out before the signal is given as input to the analog to digital converter of data acquisition. Here, an antialiasing filter is used as a Low Pass Filter (LPF). Accordingly, a second order Chebyshev LPF with a cutoff frequency of $40 \mathrm{~Hz}$ is used. Chebyshev filters differ from Butterworth filters in that they have a roll-off which is steeper and more ripple in passband (type I) or stopband (type II). Chebyshev filters have the property of minimizing the difference between real filter characteristics and the idealized one over the desired filter range, but with passband ripples. The LPF output is given to data acquisition system implemented using Arduino uno board.

\section{F. Assumptions, Measurement Protocol and Data Acquisition}

According to the BIA assumptions, the human body can be considered as a homogenous conductor having cylindrical dimensions (Fig. 10). In which bio-impedance is directly proportional to ' $\mathrm{L}$ ' and inversely proportional to 'A', where ' $\mathrm{L}$ ' is the cylinder's length and ' $\mathrm{A}$ ' is the cylinder's base crosssectional area (Fig. 10).

BIA formulation processes typically make the following assumptions for ease of calculation, though in practice the human body varies from these assumptions:

- The human body is considered as cylindrical.

- The cylindrical shape is defined by its height and weight. 


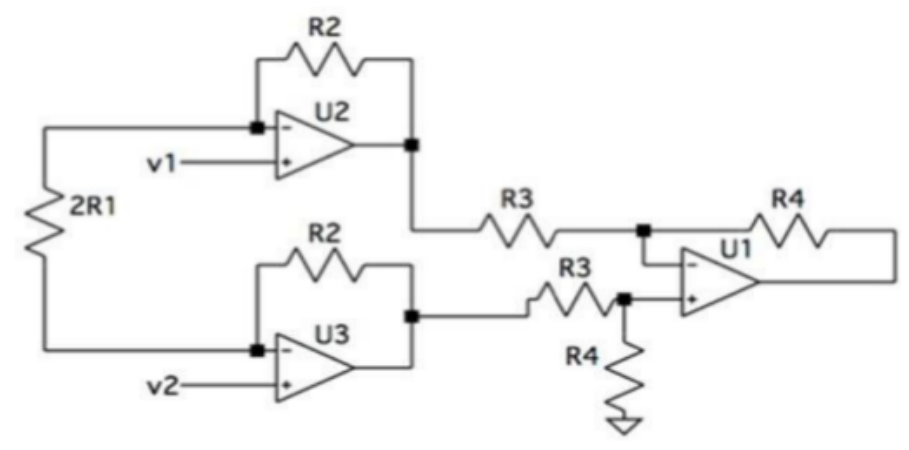

Fig. 5. Circuit Diagram for Instrumentation Amplifier.

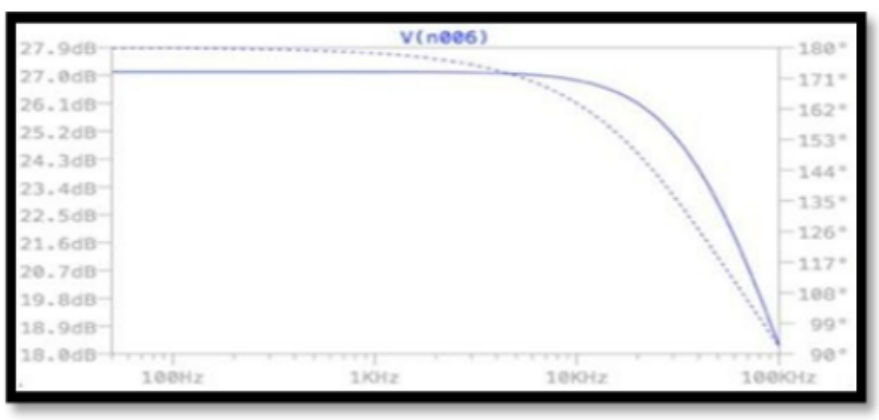

Fig. 6. Frequency Response Analysis of the Instrumentation Amplifier.

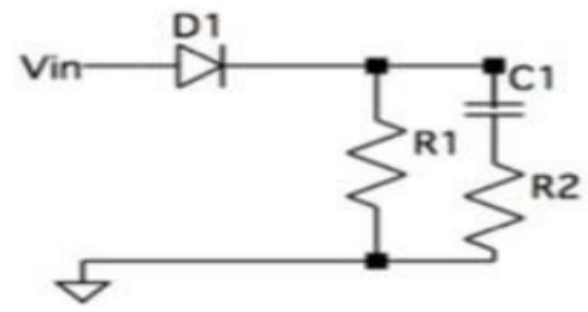

Fig. 7. Circuit Diagram of Simple Envelope Detector.

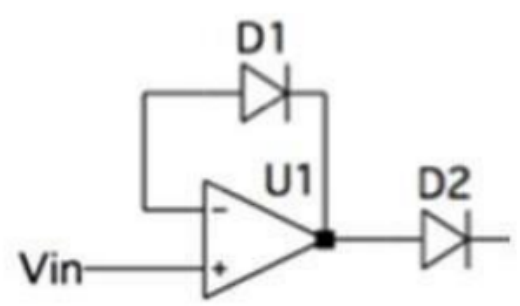

Fig. 8. Precision Detector. 


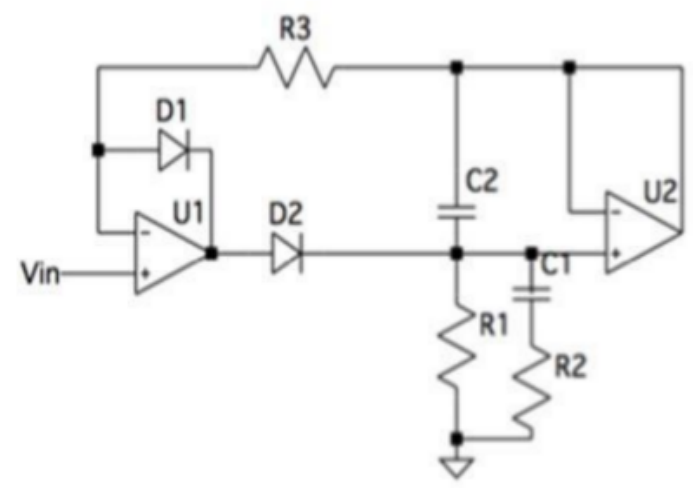

Fig. 9. Precision Envelope Detector.

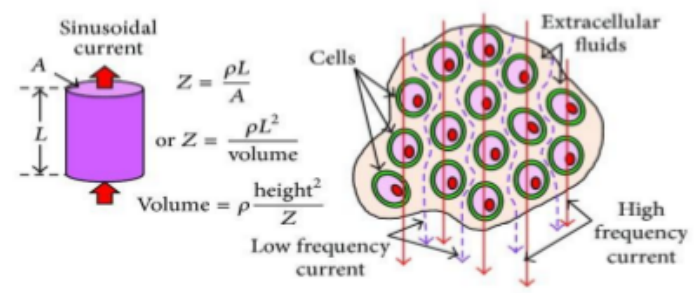

Fig. 10. The Human Body's Impedance when it is Modelled as a Homogeneous Conductor with Cylindrical Volume.

- Homogeneous and evenly distributed body composition is considered.

- The body compositions have no individual differences or variations.

- The environment parameters like temperature, other physiological parameters such as body heat or stress are assumed to be constant.

The cell membranes produce capacitive reactance due to the capacitive nature and caused by a selectively applied AC signal allows current to pass through these cell membranes using current paths largely depends upon the signal frequency (Fig. 10). The cell membranes are penetrated by current with high frequency. Thus, the current penetrates ECFs, CMs and ICFs. The low frequency current passes through ECF only as the cell membrane reactance prevents its flow through it. The BIA technique can be used to determine the (TBW) which is nothing but combination of ECW and ICW. However, it should be done at a particular frequency of the applied AC signal.

The instrument designed was tested on patients at St. Joseph hospital, Ghaziabad, India. Verbal prior approval for the study was taken telephonically from the concerned authorities. Twenty-five adult human subjects (aged 25-60 years) participated in this study. The general test setup for BIA is shown in Fig. 11. However, in our study it differed little bit for the ease of the subjects. They were made to rest in supine position for about ten to fifteen minutes. Then, $\mathrm{Ag} / \mathrm{AgCl}$ electrodes were put their right and left forearms. Volunteers were told to lie down straight and remain still during measurement.

\section{Results}

The test setup used to validate the instrument is similar to the setup used in impedance plethysmography for the cardiac output measurement. In general, impedance is a measure of resistance. Plethysmography has become the gold standard for measuring changes in blood volume in any part of the body based on electrical impedance changes [54]. It is also being used in the diagnosis of peripheral vascular diseases. In impedance plethysmography, values of instantaneous impedance $(Z)$, basal impedance $\left(Z_{0}\right)$, which is an average of calculated bio-impedance values and derivative of impedance with respect to time $(\mathrm{dZ} / \mathrm{dt})$ is used to calculate cardiac output parameters. Since the technique is a standard procedure, the values of basal impedance measured by this technique can be used to validate the instrument designed. The output of the demodulator is essentially the basal impedance of the section across which electrodes have been applied. The basal impedance values of forearm given in literature, [55] are used to validate the output signal of the instrument designed.

The output voltage signal of three sets of volunteers has been displayed in Fig. 12. The slight variations in the voltage values depict the variations in impedance $\left(Z_{0}\right)$. The basal impedance is calculated using the constant voltage level. The calculated value of basal impedance and its comparison with values of basal impedance in literature has been shown in Table I. The comparison in Table I shows that the values measured from the output of the instrument is in accordance with that of standard technique, which validates the design of the instrument. 


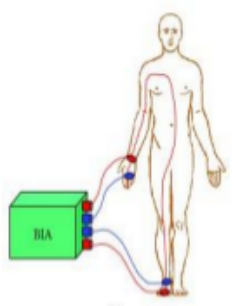

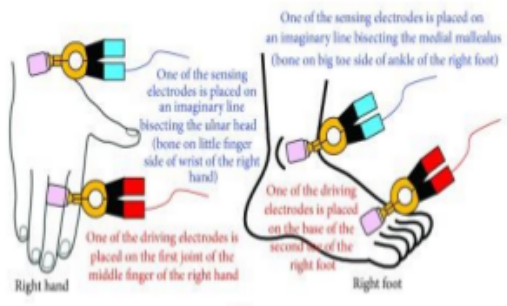

(b)

Fig. 11. General Test Setup for BIA.

TABLE I. BASAL IMPEDANCE (FOREARM)

\begin{tabular}{lllll}
\hline $\begin{array}{l}\text { SET } \\
\text { VOLUNTEERS } \\
\text { [NOs] }\end{array}$ & $\begin{array}{l}\text { MEASURED BY } \\
\text { MENT DESIGNED }\end{array}$ & INSTRU- & $\begin{array}{l}\text { STANDARD VALUES IN LIT- } \\
\text { ERATURE }\end{array}$ & \\
& & & & \\
& $\mathrm{A}_{V} \cdot \mathrm{AGE}$ & $\mathrm{A}_{V} \cdot \mathrm{Z}_{0}(\Omega)$ & AGE GROUP & $\mathrm{A}_{V} \cdot \mathrm{Z}_{0}(\Omega)$ \\
\hline $\mathrm{A}[5]$ & 40 & 58.16 & $36-45$ & $65.44 \pm 12.03$ \\
$\mathrm{~B}[8]$ & 48 & 80.57 & $46-55$ & $67.50 \pm 6.38$ \\
$\mathrm{C}[12]$ & 57 & 58.77 & $>55$ & $69.13 \pm 8.74$ \\
\hline
\end{tabular}

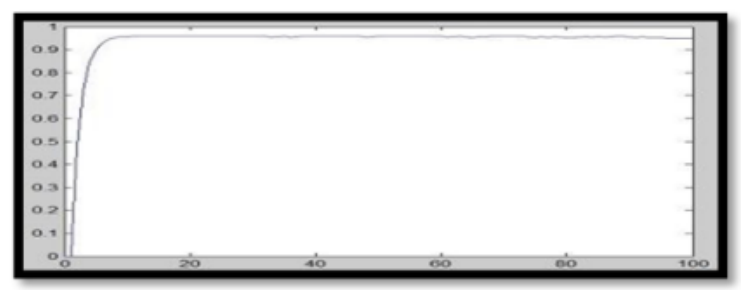

(a)

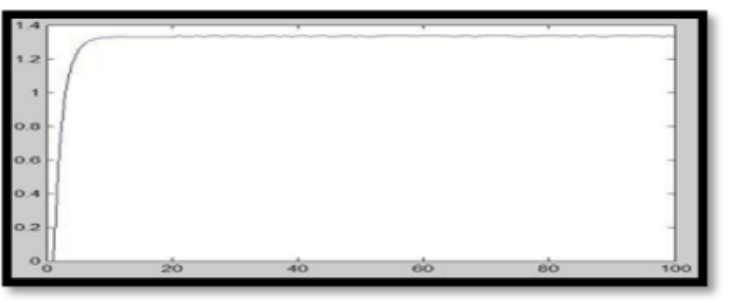

(b)

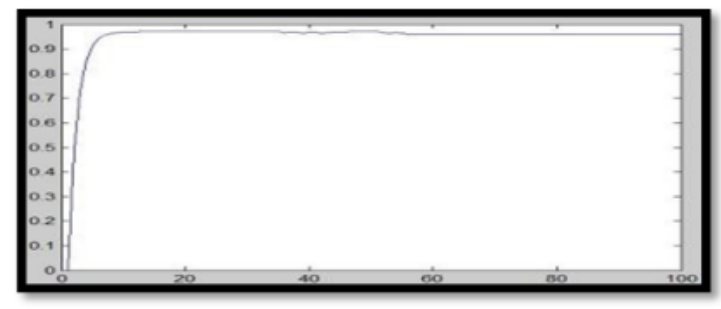

(c)

Fig. 12. Output Voltage Signals Measured using the Instrument. (a) is the Output Signal of Volunteer Set-A (b) being the Output Signal of Volunteer Set-B and (c) is the Output Signal of Volunteer Set-C. The y-axis in Each Figure is the Output Voltage Amplitude Value and the X-axis Represents Number of Samples. 


\section{CONCLUSION}

A simple low cost bio-impedance measuring instrument has been designed using common electronic blocks. The results have been validated and have been found to be accurate and reliable. Each block of the instrument has been tested using simulation and verified experimentally. The method and device developed fulfills required specifications and can be used in clinical examinations. Bio-impedance measurement techniques can prove very useful for first hand diagnosis. The major problem for using this technique for diagnosis is that its results are not standardized. By standardized one means that just by looking the graph or using its results one can say whether the results correspond to a normal person or a patient suffering from a disease. A large amount of analysis has to be done for a particular application on bio-impedance signals to enable the doctors to rely on results produced by bio-impedance analysis. The standardization of bio-impedance values for human body may be difficult due to variable ambient conditions, eating habits, lifestyle and anthropological background. So intensive and extensive research and in-depth analysis is required to be carried out towards normalization and standardization of the bio-impedance values.

Researchers in the field of bio-impedance have discovered that multifrequency bio-impedance analysis (BIA) is one of their main interests [56]. A group of researchers are exploring instrumentation and designing more advanced instrumentation. In the future, investigation and research can be carried out for Bluetooth-enabled wireless instrumentation for BIA techniques. Moreover, in future studies Bluetooth-enabled wireless instrumentation for Electrochemical Impedance Spectroscopy (EIS) techniques could be investigated. To improve cardiac health monitoring and to have potential transthoracic parameter assessment; ICG along with a bio-impedance tool with multifrequency features can be used. Furthermore, in an ambulatory or long-term monitoring requirement in the Intensive Care Unit (ICU) bio-impedance based ICG can be used. However, if future research on Bio-impedance calculation and BIA can overcome these challenges, it can be implemented more appropriately than other commonly used and popular imaging methods in specific medical diagnostic applications especially related to the imaging of brain, breast, abdominal and whole body, etc.

Bio-impedance technology is becoming more prevalent as a result of an increasing number of healthcare monitoring applications. The study presented has a wide range of implications, including the ability to improve bio-impedance studies and healthcare devices that use bio-impedance technology [7]. Bioimpedance spectroscopy measures the bio-impedance of cells and tissues covering fairly good frequency range. Physiological testing and health-monitoring systems benefit greatly from this method. Devices must be compact, wearable, or even implantable for a wide variety of applications. As a result, the next generation of bio-impedance sensing systems must be designed to save energy and resources [34]. It's critical to consider the application as well as the type of cell or tissue culture to be monitored when selecting a bio-impedance measurement technique [57]. As a result, choosing the right electrode configuration is crucial. Future research should focus on the electrodes and the bio-impedance measurement method [57]. Overall, this paper describes a low-cost, bio-electrical impedance measurement system that has been successfully developed and tested and can be used effectively and efficiently for noninvasive health monitoring. The paper also discussed some of the most important technological aspects and limitations of bio-impedance calculation and study. Finally, in this paper the theoretical dimensions, operating principles, implementations, benefits, disadvantages, and current research status, upcoming developments, and bottlenecks in bio-impedance analysis and calculation all have been covered in great detail.

\section{STATEMENTS \& DECLARATIONS}

\section{FUNDING}

The authors hereby declare that no funds, grants, or other support in any form were received during the preparation of this manuscript.

\section{COMPETING INTERESTS}

The authors have no relevant financial or non-financial interests to disclose.

\section{AUTHOR's CONTRIBUTION}

All authors contributed almost equally for material preparation, data collection and analysis to the said study related to conception and design of bio-impedance measuring instrument. Moreover, all authors read and approved the final manuscript.

\section{ETHICS APPROVAL}

Being an observational study only, it did not require any formal ethical approval. However, verbal consent from the competent authority obtained telephonically.

\section{CONSENT to PARTicipate}

No such Informed consent was required for this observational study, however individual participants if any were informed verbally.

\section{Consent to Publish}

As no figure in the manuscript depicts human participants, therefore no such consent for publication of the images of the human research participants is required.

\section{REFERENCES}

[1] F. H. Netter, "Atlas of Human Anatomy, Rittenhouse. 2nd. Book Distributors Inc,” 1997, [Google Scholar].

[2] J. J. Ackmann, "Complex bioelectric impedance measurement system for the frequency range from $5 \mathrm{~Hz}$ to $1 \mathrm{MHz}$,' Annals of Biomedical Engineering, vol. 21, no. 2, pp. 135-146, 1993, [PubMed] [Google Scholar].

[3] K. Cha, G. M. Chertow, J. Gonzalez, J. M. Lazarus, and D. W. Wilmore, "Multifrequency bioelectrical impedance estimates the distribution of body water," Journal of Applied Physiology, vol. 79, no. 4, pp. 13161319, 1995, [PubMed] [Google Scholar].

[4] A. Roy et al., "An experimental method of bioimpedance measurement and analysis for discriminating tissues of fruit or vegetable," AIMS Biophysics, vol. 7, no. 1, pp. 41-53, 2020.

[5] S. Grimnes and G. Martinsen, "Academic Press, Bioimpedance \& Bioelectricity Basics," 2021. 
[6] L. Jodal, "MSc, Medical Physicist, lecture notes on the electrical theory behind the measurement of body fluids with bioimpedance spectroscopy (BIS)," 2021.

[7] B. Louarroudi and Sanchez, "On the correct use of stepped-sine excitations for the measurement of time-varying bioimpedance," Physiological Measurement, vol. 38, no. 2, pp. N73-N80, 2017.

[8] J. W. Haslett and M. K. N. Rao, "A High Quality Controlled Current Source," in Instrumentation and Measurement," IEEE Transactions on, vol. 28, no. 2, pp. 132-140, 1979.

[9] P. Annus, A. Krivoshei, M. Min, and T. Parve, "Excitation Current Source for Bioimpedance Measurement Applications: Analysis and Design," Instrumentation and Measurement Technology Conference Proceedings, pp. 848-863, 2008.

[10] R. Pallas-Areny and J. G. Webster, “AC Instrumentation,” 2021.

[11] "Electrical Cardiometry" Wikipedia," 2021.

[12] "Respiration rate measurement based on impedance pneumography Application report, SBAA1811-February 2011, Texas Instruments," 2021

[13] O. G. Martinsen, "Utilizing characteristic electrical properties of the epidermal skin layers to detect fake fingers in biometric fingerprint systems-A pilot study," Biomedical Engineering, vol. 54, pp. 891-894, 2007.

[14] D. Naranjo-Hernández et al., "Fundamentals, Recent Advances, and Future Challenges in Bioimpedance Devices for Healthcare Applications," Journal of Sensors, Hindawi, 2019, Article ID 9210258.

[15] P. S. H. Jose et al., "A Non-Invasive Method for Measurement of Blood Glucose using Bio Impedance Technique," In 2nd International Conference on Signal Processing and Communication (ICSPC), 2019, IEEE.

[16] J. Nyboer, "Electrical impedance plethysmography; a physical and physiologic approach to peripheral vascular study," Circulation, vol. 2, no. 6, pp. 811-821, 1950, [PubMed] [Google Scholar].

[17] R. W. Griffiths, M. E. Philpot, B. J. Chapman, and K. A. Munday, "Impedance cardiography: non-invasive cardiac output measurement after burn injury," International Journal of Tissue Reactions, vol. 3, no. 1, pp. 47-55, 1981, [PubMed] [Google Scholar].

[18] C. J. Schuster and H. P. Schuster, "Application of impedance cardiography in critical care medicine," Resuscitation, vol. 11, no. 3-4, pp. 255-274, 1984, [PubMed] [Google Scholar].

[19] "Internet Article, JAWON Medical, South Korea," 2021, http://www.ja won.co.kr/eng/technology/body-composition/principles- of-bia.php.

[20] "Internet Article. Bodystat Limited, USA," 2021, http://www.bodystat. com/science.

[21] D. Miklavcic, N. Pavselj, and F. X. Hart, "Electric Properties of Tissues," Wiley Encyclopedia of Biomedical Engineering, 2006, [Google Scholar].

[22] H. P. Schwan, "Electrical properties of tissue and cell suspensions: Mechanisms and models," Proc IEEE Adv Biol Med Soc, vol. 1, pp 1:A70-A1, 2002, [Google Scholar].

[23] R. Pethig and D. B. Kell, "The passive electrical properties of biological systems: Their significance in physiology, biophysics and biotechnology," Phys Med Biol, vol. 32, no. 933, 1987, [PubMed] [CrossRef] [Google Scholar].

[24] U. G. Kyle, I. Bosaeus, D. L. AD, and A. D, "Bioelectrical impedance analysis part I: review of principles and methods," Clinical Nutrition, vol. 23, pp. 1226-43, 2004, [PubMed] [CrossRef] [Google Scholar].

[25] S. Grimnes and $\varnothing$. G. Martinsen, "Bioimpedance \& Bioelectricity Basics. Elsevier Science," 2014, 3rd ed. [Google Scholar].

[26] K. Asami, "Characterization of heterogeneous systems by dielectric spectroscopy," Prog Polym Sci, vol. 27, pp. 1617-59, 2002, [CrossRef] [Google Scholar].

[27] K. Heileman, J. Daoud, and M. Tabrizian, "Dielectric spectroscopy as a viable biosensing tool for cell and tissue characterization and analysis," Biosens Bioelectron, vol. 49, pp. 348-59, 2013, [PubMed] [CrossRef] [Google Scholar].

[28] P. J. Riu and C. On, "Bioelectrical parameters of the whole human body obtained through bioelectrical impedance analysis," Bioelectromagnetics, vol. 25, pp. 69-71, 2004, [PubMed] [CrossRef] [Google Scholar].
[29] C. Gabriel, S. Gabriel, and E. Corthout, "The dielectric properties of biological tissues: I. Literature survey," Phys Med Biol, vol. 41, pp. 2231-49, 1996, [PubMed] [CrossRef] [Google Scholar].

[30] Ø. G. Martinsen, S. Grimnes, and H. P. Schwan, "Interface phenomena and dielectric properties of biological tissue," Encyclopedia of Surface and Colloid Science, vol. 20, pp. 2643-53, 2002, [Google Scholar].

[31] D. A. Dean, T. Ramanathan, D. Machado, and R. Sundararajan, "Electrical Impedance Spectroscopy Study of Biological Tissues," J Electrostat, vol. 66, no. 3-4, pp. 165-77, 2008, [PMC free article] [PubMed] [CrossRef] [Google Scholar].

[32] H. Kwon, A. L. Mcewan, T. I. Oh, A. Farooq, E. J. Woo, and J. K. Seo, "A local region of interest imaging method for electrical impedance tomography with internal electrodes," Comput Math Methods Med, vol. 9, 2013, [PMC free article] [PubMed] [CrossRef] [Google Scholar].

[33] J. K. Seo, T. K. Bera, H. Kwon, and R. J. Sadleir, "Effective Admittivity of Biological Tissues as a Coefficient of Elliptic PDE," Computational and Mathematical Methods in Medicine, vol. 2, 2013, [PMC free article] [PubMed] [CrossRef] [Google Scholar].

[34] F. Soulier et al., "Very Low Resource Digital Implementation of Bioimpedance Analysis," Sensors (Basel), MDPI, vol. 19, p. 3381, 2019.

[35] J. Ojarand et al., "How many frequencies to use in electrical bioimpedance measurements," in Impedance Spectroscopy. Advanced Applications: Battery Research, Bioimpedance, System Design. Publisher: Walter de Gruyter, 2018, pp. 161-168.

[36] S. F. Khalil, M. S. Mohktar, and F. Ibrahim, "The Theory and Fundamentals of Bioimpedance Analysis in Clinical Status Monitoring and Diagnosis of Diseases," A Review. Sensors, vol. 14, 2014, [PMC free article] [PubMed] [Google Scholar].

[37] R. Kusche et al., "Dry electrodes for bioimpedance measurements - Design, characterization and comparison," Biomedical Physics \& Engineering Express, IOP, vol. 5, no. 1, 2018.

[38] H. Kalvøy, L. Frich, S. Grimnes, and Ø. G. Martinsen, "Impedancebased tissue discrimination for needle guidance," Physiological Measurements, vol. 30, 2009, [PubMed] [Google Scholar].

[39] A. Yúfera and A. Rueda, "A Method for Bioimpedance Measure with Four- and Two-Electrode Sensor Systems," in 30th Annual International IEEE EMBS Conference Vancouver. British Columbia, Canada, 2008, [PubMed] [CrossRef] [Google Scholar].

[40] X. Huang, "Simulation of Microelectrode Impedance Changes Due to Cell Growth," IEEE Sensors Journal, vol. 4, pp. 576-83, 2004, [CrossRef] [Google Scholar].

[41] I. Giaever, "Use of Electric Fields to Monitor the Dynamical Aspect of Cell Behavior in Tissue Cultures," IEEE Transaction on Biomedical Engineering, vol. 33, pp. 242-7, 1986, BME. [PubMed] [CrossRef] [Google Scholar].

[42] T. S. Carvalho, A. L. Fonseca, A. Coutinho, B. Jotta, A. V. Pino, and M. N. Souza, "Comparison of bipolar and tetrapolar techniques in bioimpedance measurement. XXIV Congresso Brasileiro de Engenharia Biomédica - CBEB," 2014, [Google Scholar].

[43] L. A. Geddes and R. Roeder, "Criteria for the selection of materials for implanted electrodes," Annals of Biomedical Engineering, vol. 31, no. 7, pp. 879-90, 2003, [PubMed] [CrossRef] [Google Scholar].

[44] H. Kalvøy, G. K. Johnsen, Ø. G. Martinsen, and S. Grimnes, "New Method for Separation of Electrode Polarization Impedance from Measured Tissue Impedance," The Open Biomedical Engineering Journal, vol. 5, pp. 8-13, 2011, [PMC free article] [PubMed] [CrossRef] [Google Scholar].

[45] J. Wang, W. Hu, T. Kao, C. Liu, and S. Lin, "Development of forearm impedance plethysmography for the minimally invasive monitoring of cardiac pumping function," Journal of Biomedical Science and Engineering, vol. 4, pp. 122-129, 2011, Inter instrument comparison of bioimpedance spectroscopic analyzers.

[46] A. S. Sedra and K. C. Smith, "Microelectronic Circuits," 2021, 5th edition Oxford International Student addition.

[47] "Bioelectrical impedance analysis in body composition measurement: National institutes of health technology assessment conference statement," 1994.

[48] G. B. Clayton, "88 Practical Opamp Circus,” 2021, 5th edition Oxford International Student addition. 
[49] "ICL 8038 datasheet," 2021.

[50] N. Neshatvar et al., "Analog Integrated Current Drivers for Bioimpedance Applications: A Review," Sensors (Basel), vol. 19, no. 4 p. 756, 2019, Pub Med.

[51] S. I. Kim and T. S. Suh, "Current Source for Wideband Electrical Bioimpedance Spectroscopy Based on a Single Operational Amplifier," in World Congress of Medical Physics and Biomedical Engineering. Springer-Verlag, 2006, 20006COEX Seoul, Korea. Berlin Heidelberg: Springer-Verlag., 2007.

[52] "Biopotential Amplifiers in The Biomedical Engineering HandBook," 2000, by Joachim H. Nagel edited by Joseph D. Bronzino.

[53] M. . Qu, Y. Zhang, J. G. Webster, and W. J. Tompkins, "Motion Artifact from Spot and Band Electrodes During Impedance Cardiography," Biomedical Engineering, no. 11, pp. 1029-1036, 1986.
[54] “25 years of impedance plethysmography," 2003, BARC Newsletter No.236.

[55] D. L. Prajapati, V. Chintan, Parmar, A. Pradnya, Gokhale, B. Hemant, C. J. S. Mehta, and Non, "Invasive Assessment of Blood Flow Index In Healthy Volunteers Using Impedance Plethysmography," International Journal of Medical and Health Sciences, 2013, ISSN 2277-4505.

[56] B. T. Kanti, "Bioelectrical Impedance Methods for Non-invasive Health Monitoring: A Review," J Med Eng, p. 381251, 2014, PMCID: PMC4782691, PMID: 27006932.

[57] M. Amini, J. Hisdal, and H. Kalvøy3, "Applications of Bioimpedance Measurement Techniques in Tissue Engineering," J. Electrical Bioimpedance, vol. 9, no. 1, pp. 142-158, 2018. 